\title{
REVISED Inducible TDG knockout models to study epigenetic
}

\section{regulation [version 2; peer review: 3 approved]}

\author{
Simon D. Schwarz (D1), Eliane Grundbacher'1, Alexandra M. Hrovat (D1), \\ Jianming Xu¹ , Anna Kuśnierczyk², Cathrine B. Vågbø², Primo Schär(D1, \\ David Schuermann (iD)
}

\footnotetext{
${ }^{1}$ Department of Biomedicine, University of Basel, Basel, 4058, Switzerland

2Proteomics and Modomics Experimental Core Facility (PROMEC), Norwegian University of Science and Technology, Trondheim, 7491, Norway
}

\author{
V2 First published: 09 Sep 2020, 9:1112 \\ https://doi.org/10.12688/f1000research.25637.1 \\ Latest published: 19 Oct 2020, 9:1112 \\ https://doi.org/10.12688/f1000research.25637.2
}

\begin{abstract}
Mechanistic and functional studies by gene disruption or editing approaches often suffer from confounding effects like compensatory cellular adaptations generated by clonal selection. These issues become particularly relevant when studying factors directly involved in genetic or epigenetic maintenance. To provide a genetic tool for functional and mechanistic investigation of DNA-repair mediated active DNA demethylation, we generated experimental models in mice and murine embryonic stem cells (ESCS) based on a minigene of the thymine-DNA glycosylase (TDG). The loxP-flanked miniTdg is rapidly and reliably excised in mice and ESCs by tamoxifen-induced Cre activation, depleting TDG to undetectable levels within 24 hours. We describe the functionality of the engineered miniTdg in mouse and ESCS (TDGiKO ESCS) and validate the pluripotency and differentiation potential of TDGiKO ESCS as well as the phenotype of induced TDG depletion. The controlled and rapid depletion of TDG allows for a precise manipulation at any point in time of multistep experimental procedures as presented here for neuronal differentiation in vitro. Thus, we provide a tested and well-controlled genetic tool for the functional and mechanistic investigation of TDG in active DNA (de)methylation and/or DNA repair with minimal interference from adaptive effects and clonal selection.
\end{abstract}

Keywords

Embryonic Stem Cells, TDG, Active DNA Demethylation, Base Excision Repair, Neuronal Differentiation, Minigene, Cre/loxP, Tamoxifen

\section{Open Peer Review}

Approval Status
$\begin{aligned} & \text { version 2 } \\ & \text { (revision) } \\ & \text { 19 Oct 2020 }\end{aligned}$
version 1
09 Sep 2020

1. Jonathan Sczepanski, Texas A\&M University, College Station, USA

2. Joseph Torchia, Western University, London, Canada

\section{Sebastian Bultmann (iD), Ludwig-}

Maximilians-Universität München, Munich, Germany

Any reports and responses or comments on the article can be found at the end of the article. 
Corresponding authors: Primo Schär (primo.schaer@unibas.ch), David Schuermann (david.schuermann@unibas.ch)

Author roles: Schwarz SD: Conceptualization, Formal Analysis, Investigation, Methodology, Project Administration, Visualization, Writing - Original Draft Preparation, Writing - Review \& Editing; Grundbacher E: Investigation, Methodology, Writing - Review \& Editing; Hrovat AM: Investigation, Writing - Review \& Editing; Xu J: Investigation, Writing - Review \& Editing; Kuśnierczyk A: Investigation, Resources, Writing - Review \& Editing; Vågbø CB: Investigation, Resources, Writing - Review \& Editing; Schär P: Conceptualization, Funding Acquisition, Project Administration, Resources, Supervision, Writing - Review \& Editing; Schuermann D: Conceptualization, Formal Analysis, Investigation, Methodology, Project Administration, Supervision, Writing - Review \& Editing

Competing interests: No competing interests were disclosed.

Grant information: This work was supported by the Swiss National Science Foundation, grant 156467.

Copyright: ( 2020 Schwarz SD et al. This is an open access article distributed under the terms of the Creative Commons Attribution License, which permits unrestricted use, distribution, and reproduction in any medium, provided the original work is properly cited.

How to cite this article: Schwarz SD, Grundbacher E, Hrovat AM et al. Inducible TDG knockout models to study epigenetic regulation [version 2; peer review: 3 approved] F1000Research 2020, 9:1112 https://doi.org/10.12688/f1000research.25637.2

First published: 09 Sep 2020, 9:1112 https://doi.org/10.12688/f1000research.25637.1 


\section{REVISED Amendments from Version 1}

In response to the reviewers' comments and suggestions, the article has been revised as follows:

The integration of the miniTdg transgene in a random genomic location was clarified.

Indicators of statistical significance and extended description of the statistical approaches were added to all figures and respective legends.

Off-target analyses for the two ESC clones generated by CRISPR/ Cas9 were added to the Extended data.

Clarifications and text improvement were made according to the comments and questions raised by the reviewers, referring to the generation and use of the engineered system in cultured cells and animals as well as to technical details.

Typos and ambiguous phrasing were corrected throughout the article.

Any further responses from the reviewers can be found at the end of the article

\section{Introduction}

To allow for the differentiation to a multitude of cell types during the development of multicellular organisms, stem cells need to respond to a variety of extrinsic and intrinsic developmental cues and integrate these into epigenetically stabilized gene expression patterns during lineage specification. The underlying mechanisms to ensure this necessary epigenetic plasticity are therefore active and highly dynamic in embryonic stem cells (ESC). One of those mechanisms includes the thymine DNAglycosylase (TDG) and the downstream factors of DNA base excision repair (BER), which were shown to be involved in the dynamic, locus-specific regulation of DNA methylation. Following the activity of dioxygenases of the ten-eleven-translocation (TET) family that iteratively oxidize 5-methylcytosine (5-mC) to formyl- and carboxylcytosine (5-fC, 5-caC), TDG-induced BER provides a mechanism of active DNA demethylation and, thereby, impact gene regulation (Cortázar et al., 2011; Cortellino et al., 2011; He et al., 2011; Ito et al., 2011; Schuermann et al., 2016; Shen et al., 2013; Weber et al., 2016). Studying the precise function of TDG (and BER) in epigenetic regulation during differentiation of stem cells, however, is challenging due to its multiple and interwoven interactions with other proteins like transcription factors and epigenetic modifiers (Henry et al., 2016; Jacobs \& Schär, 2012; Léger et al., 2014), the deregulation of which may cause a plethora of confounding effects. Also, genetic depletion of TDG itself is not compatible with embryonic development (Cortázar et al., 2011; Cortellino et al., 2011). To reduce and circumvent such confounders in functional studies of TDG (and BER) in murine ESCs, we established and validated a $T d g$ minigene, introduced it into mice and derived a series of different ESC variants. The minigene carries, among other features, a loxP-flanked coding region of $T d g$, allowing for a controlled and fast, 4-hydroxytamoxifen (OHT)-inducible depletion of TDG in the background of a homozygous disruption of the endogenous $T d g$. By eliminating phenotypic divergence between TDG proficient and deficient ESCs, this versatile model facilitates precise functional and mechanistic investigations into TDG-mediated active DNA demethylation. This novel $T d g$ minigene introduced into mice as well as ESCs will facilitate future research in the field of active DNA demethylation in vivo and in vitro.

\section{Methods}

Ethics statement and Animal Work

All animal work was carried out in accordance with the Swiss Animal Welfare Act and the guidelines of the Swiss Federal Veterinary Office (SFVO) or with the UK Animals (Scientific Procedures) Act. Housing, breeding and experimentation of mice has been performed with the approval of the Cantonal Veterinary Office of Basel-Stadt (Licenses 10062-H, 1912) or was covered by a project license to Wolf Reik (80/1896) further regulated by the Babraham Institute Animal Welfare, Experimentation, and Ethics Committee. Mice were housed under specific pathogen free condition in individually ventilated cages under $12 \mathrm{~h} \mathrm{light/dark}$ cycles at $22 \pm 2^{\circ} \mathrm{C}$ and $35-65 \%$ relative humidity. Animal and colony health was checked daily and quarterly, respectively. Sterilized diet (Kliba-Nafag Extrudate 3436) and water was provided ad libitum.

$T d g^{\text {tmlPsch }}$ mice (http://www.informatics.jax.org/allele/MGI:5487834) were generated from E14 ESC (RRID:CVCL_C320) and backcrossed for more than 10 generations with female C57BL/ 6JRj mice, obtained at 7-8 weeks of age from Janvier Labs. A $T d g$ expression cassette (pTCO2-mTDGi.0, Addgene Plasmid \#149429) was introduced as single copy into C57BL/6JRj mice by pronuclear injection and crossed into $T d g^{t m l P s c h}$ mice. Genotyping was done by PCR with the HOT FIREPol (Solis BioDyne) on diluted crude DNA preparations (diluted by a factor of five with $10 \mathrm{mM}$ Tris- $\mathrm{HCl} \mathrm{pH} 8$ ) by boiling toe clips with $25 \mathrm{mM} \mathrm{NaOH} / 0.2 \mathrm{mM}$ EDTA for $1 \mathrm{~h}$ followed by neutralization with $40 \mathrm{mM}$ Tris-HCl. Reactions were performed according to the manufacturer's recommendation with 35 PCR cycles at $95^{\circ} \mathrm{C}$ for $30 \mathrm{~s}, 60^{\circ} \mathrm{C}$ for $30 \mathrm{~s}$, and at $72^{\circ} \mathrm{C}$ for $60 \mathrm{~s}$ (for details about all used primers and annealing temperatures in PCR reactions, see Extended data Table S1) (Schwarz, 2020).

\section{Generation of ESCS}

ESCs were derived from male blastocysts from timed matings of male miniTdg $g^{\text {tg } / g} / / T d g^{-/}$mice with 2 super-ovulated female $\mathrm{Td}^{+/-}$mice and cultured on murine immortalized feeder cells in $2 \mathrm{i}$ medium with leukemia inhibitory factor LIF (MerckMillipore) (Ying et al., 2008).

To introduce the inducible Cre recombinase into the ROSA26 locus, $30 \mu \mathrm{g}$ of the targeting vector (pROSA26-ERT2CreERT2, Addgene Plasmid \#149436) was electroporated into $15 \times 10^{6}$ mESCs using a gene pulser Xcell (Bio-Rad) at $240 \mathrm{~V}$ and $475 \mu \mathrm{F}$. Transgenic cells were selected with 8 and $5 \mu \mathrm{g} / \mathrm{ml}$ blasticidin for a week each, before colonies were picked and then amplified without selection pressure. From blasticidin-resistant colonies, genomic DNA was extracted with the "QIamp DNA mini" kit (Qiagen) and screened for a targeted integration at the 3' junction of the ROSA26 locus by PCR with the Phusion polymerase (NEB) (see Extended data, Table S1) (Schwarz, 2020), applying a general three-step cycling protocol: initial denaturation $95^{\circ} \mathrm{C}$ for $30 \mathrm{~s}, 35$ times $95^{\circ} \mathrm{C}$ for $10 \mathrm{~s}$, annealing for 
$20 \mathrm{~s}, 72^{\circ} \mathrm{C}$ for $15-80$ s. PCR reactions were analyzed by gel electrophoresis followed by image acquisition with a U:Genius 3 system (Version 3.0.12.0, Syngene).

To evoke a disruption of the $N e o^{R}$ reading frame by CRISPR/ Cas9, two guide RNAs $\left(\left(\mathrm{Neo}^{\mathrm{R}}\right)\right.$ 3: 5'-GCCGATCCCATATTGGC TGCAGG-3', $\left(\mathrm{NeO}^{\mathrm{R}}\right)$ 4: 5'-GAAGGCGATGCGCTGCGAATCG G-3', IDT) were designed and transfected with TransIT-X2 (Mirus Bio) into the TDGiKO1 ESCs. RNP assembly was performed according to the manufacturers protocol (IDT). One day after transfection, single cells were sorted with a FACSaria IIIu (BD BioSciences) into 96-well plates coated with inactivated mouse fibroblasts, based on GFP expression from a co-transfected pEGFP-N1 plasmid (Clontech). Screening for the successful disruption of the $N e o^{R}$ gene was done by PCR as described above and assaying the loss of the neomycin resistance with the Cell Counting Kit-8, according to the manufacturer's instructions (CCK-8, Dojindo).

\section{Cell culture and differentiation}

ESCs were cultured under a controlled atmosphere $\left(37^{\circ} \mathrm{C}, 5 \%\right.$ $\mathrm{CO}_{2}, 95 \%$ humidity) in serum-free $2 \mathrm{i}$ medium with $1000 \mathrm{U} / \mathrm{ml}$ LIF, without antibiotics unless indicated otherwise. The 4-OHT (Sigma-Aldrich H7904) was dissolved in DMSO (stock $10 \mathrm{mM}$ ) and administered at indicated concentrations for $2 \mathrm{~h}$.

Neuronal differentiation was performed as described before (Bibel et al., 2007; Cortázar et al., 2011; Steinacher et al., 2019). Differentiation towards cardiomyocytes was performed by culturing ESCs in classical ESC medium (ESM) with $15 \%$ FCS without LIF in non-adhesive petri dishes (Greiner) for at least 10-14 days.

All pictures were taken with a DM IL LED Fluo microscope and MC170 HD camera (Leica) at 100x magnification.

\section{Molecular analysis of ROSA26 targeting and miniTdg excision}

Probes for Southern blotting were generated by PCR amplification with Taq DNA Polymerase (Qiagen) from the ROSA26 targeting plasmid using the DIG DNA labeling mix (Roche). Southern blotting was done with $20 \mu \mathrm{g}$ of genomic DNA, extracted by the "Genomic tip 100G" kit (Qiagen). Probes were hybridized according to the "DIG Application Manual for Filter Hybridization" by Roche. Signals were acquired by exposing the membrane to chemiluminescence detection films (Amersham/ GE Healthcare) and digitalized by a CanoScan 8400F scanner with CanoScan Toolbox (Version 4.9.3).

Excision of the miniTdg gene by Cre was assessed by qPCR with the "Rotor-Gene SYBR Green PCR Kit" (Qiagen) on a Rotor Gene 3000 (Qiagen) system. After $95^{\circ} \mathrm{C}$ for $5 \mathrm{~min}$, qPCR reactions followed a two-step cycling $(40 \mathrm{x})$ protocol: $95^{\circ} \mathrm{C}$ for $5 \mathrm{~s}$, $60^{\circ} \mathrm{C}$ for $30 \mathrm{~s}$, terminated by ramping from 60 to $95^{\circ} \mathrm{C}$ in $175 \mathrm{~s}$ to generate a melting curve. PCRs to exclude background recombination by Cre were done with NEB Phusion polymerase as described above.

\section{Gene expression analyses}

Total RNA was extracted with the RNAeasy Mini Kit (Qiagen), including on-column DNase digest, and reverse transcribed with RevertAid First Strand cDNA synthesis kit (ThermoFisher) using oligo-dT primers. qPCR was performed as described above with marker-specific primers (see Extended data, Table S1) (Schwarz, 2020).

Protein levels were analyzed by western blotting of $50 \mu \mathrm{g}$ of NP-40 (or SDS) whole-cell extracts, separated by SDSPAGE, onto a nitrocellulose membrane (Amersham) and immunodetection with anti-mTDG antibody (rabbit polyclonal L58, Lab P.Schär, dilution $1: 10^{\prime} 000,1 \mathrm{~h}$ at $33^{\circ} \mathrm{C}$ ) and anti-GAPDH antibody (Sigma-Aldrich Cat\# G9545, RRID:AB_796208 dilution 1:20'000, $1 \mathrm{~h}$ at RT). Chemiluminescence signals were detected with a Fusion FX7 system (Software version 16.15.0.0, Vilber).

\section{Detection of oxidized variants of 5-methylcytosine}

DNA was extracted and purified with the Genomic tip 100G Kit (Qiagen). High-performance liquid chromatographytandem mass spectrometry analysis was performed on $10 \mu \mathrm{g}$ of genomic DNA as described before (Weber et al., 2016).

\section{Statistical analysis}

To test for statistical significance ( $p \leq 0.05$ ), we performed two-tailed Student's $t$-tests on the indicated number of replicates in Microsoft Excel (Version 16.0.4954.1000)

\section{Results and discussion}

Generation of a chimeric Tdg minigene and derivation of murine ESCs carrying a tamoxifen-inducible Cre recombinase

To facilitate genetic manipulation of the $T d g$ gene $(\sim 28 \mathrm{~kb}$ including the putative promoter), we first constructed a synthetic $T d g$ minigene (miniTdg, $\sim 11 \mathrm{~kb}$ ) (Figure 1A). The minigene consists of the endogenous $T d g$ promoter and terminator sequences flanking the $T d g$ coding sequence (CDS). It also includes the rabbit $\beta$-globin intron at the authentic position of the first intron of the $T d g$ transcript variant 2 (Genebank NM_172552.4). This intron allows for the expression of the two naturally occurring $T d g$ splice variants (Um et al., 1998). Inaddition, the miniTdg contains two loxP sites in the same orientation for Cre-recombinase-mediated excision of the codingregion. A plasmid carrying the miniTdg gene (pTCO2-mTDGi.0) was introduced into C57BL/6 mice at a random genomic location by pronuclear injection and offspring carrying the minigene were crossed into heterozygous $T d g^{\text {tmlPsch }}$ mutant mice (Kunz et al., 2009). When breedingheterozygous mice for the miniTdg transgene and the $T d g$ $\mathrm{KO}$ allele, the genotypes of offspring followed the expected Mendelian pattern for two loci (Figure 1B). The miniTdg allele segregated with a $3: 1$ ratio, consistent with a random integration into a single genomic locus. About $25 \%$ of born mice with the miniTdg gene were homozygous for the $T d g^{\mathrm{tm} 1 \mathrm{Psch}} \mathrm{KO}$ allele while no homozygous $T d g \mathrm{KO}$ mouse was obtained without the transgene. This shows that the miniTdg transgene is fully 
A

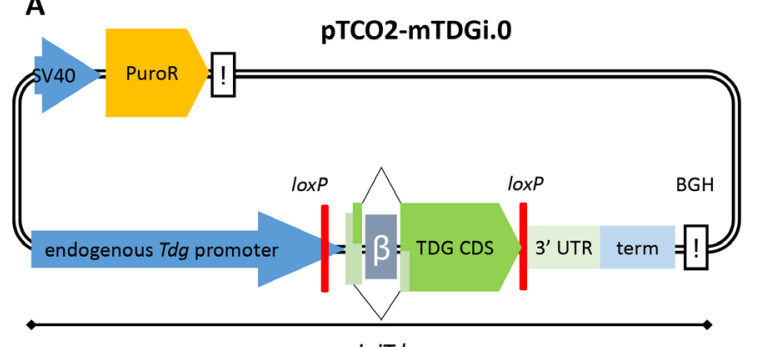

B

\begin{tabular}{|c|c|c|c|c|c|}
\hline \multicolumn{6}{|c|}{ miniTdg ${ }^{t g /+} / /$ Tdg $^{-/+} \times$miniTdg $t g /+/ /$ Tdg $^{-/+}$} \\
\hline \multicolumn{2}{|c|}{ Genotype } & \multicolumn{2}{|c|}{ Expected } & \multicolumn{2}{|c|}{ Obtained } \\
\hline$T d q$ & miniTdq & $\underline{T d q}$ & miniTdq & $\underline{T d q}$ & miniTdq \\
\hline$-/-$ & \multirow{3}{*}{ - } & 7 & \multirow{3}{*}{29} & 0 & \multirow{3}{*}{28} \\
\hline$+/-$ & & 15 & & 15 & \\
\hline$+/+$ & & 7 & & 13 & \\
\hline$-/-$ & \multirow{3}{*}{+} & 22 & \multirow{3}{*}{88} & 22 & \multirow{3}{*}{88} \\
\hline$+/-$ & & 44 & & 38 & \\
\hline$+/+$ & & 22 & & 28 & \\
\hline
\end{tabular}

C

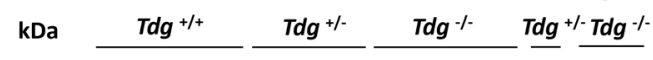

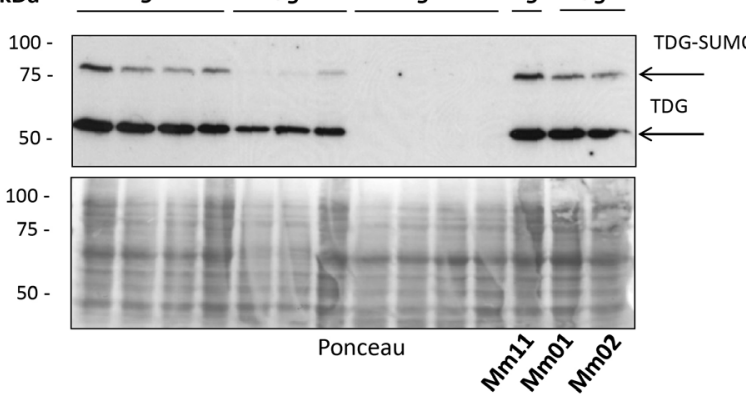

D

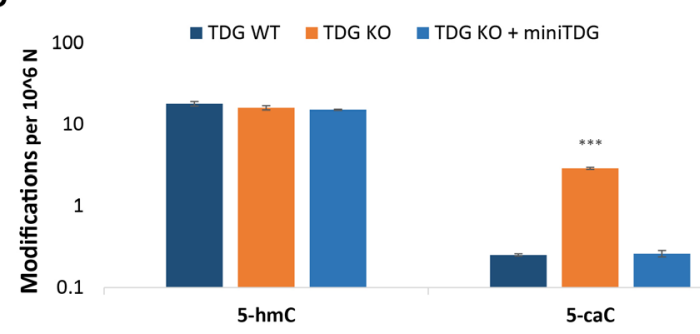

$\mathbf{E}$

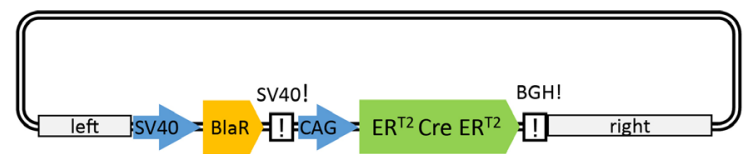

$\mathbf{F}$
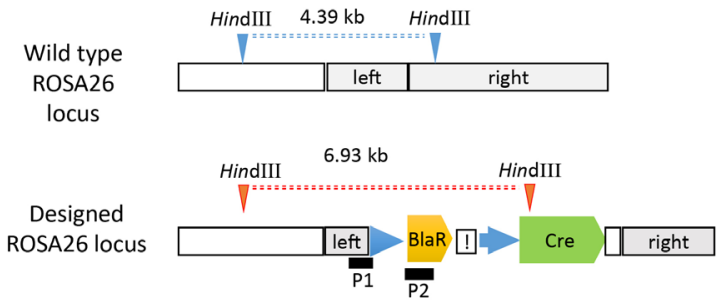
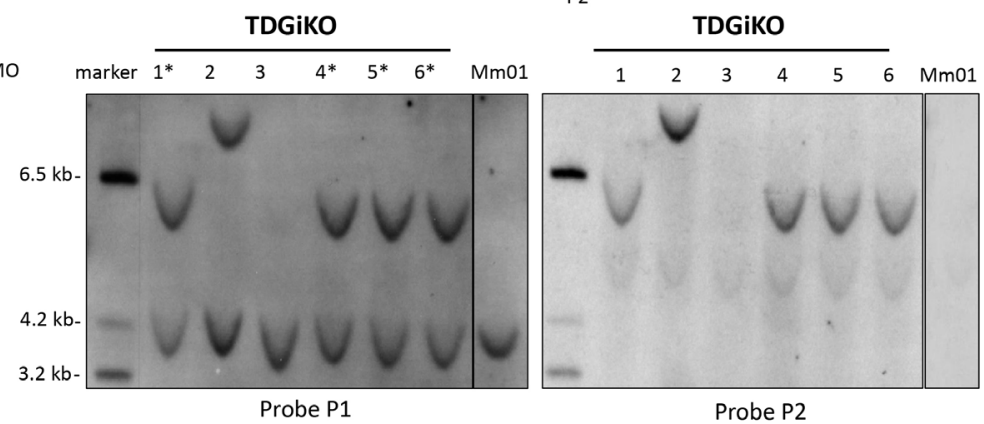

Figure 1. miniTdg complementation of $\boldsymbol{T d}^{-/-}$mice and $\mathbf{m E S C s}$. (A) Synthetic miniTdg gene on the plasmid integrated to a random genomic locus by pronuclear injection. miniTdg consisting of $6 \mathrm{~kb}$ endogenous Tdg promoter, the Tdg coding sequence (CDS) including a chimeric splice donor (Tdg exon 1 of transcript variant 2 NM_172552.4, rabbit $\beta$-globin intron), to allow for alternative splicing, 3 kb of the $3^{\prime}$ untranslated region ( $3^{\prime}$ UTR) and terminator (term) of $T d \bar{g}$, and the bovine growth hormone terminator sequence (BGH!). loxP sites are indicated in red. (B) Expected and obtained genotype distribution in offspring $(n=116)$ from crosses of heterozygous mice. (C) Top: Immunoblot for TDG of whole-cell SDS-extracts from derived ESCS with different genotype. Bottom: Loading control by Ponceau staining of the membrane. (D) Accumulation of oxidized 5-mC derivates measured by HPLC-MS/MS in ESCs with the indicated genotype. For each genotype, means and standard deviations of two independent clones with 1 or 2 repeated measurements are shown. Asterisks indicate $p$-values of Student's t-test, compared to TDG WT: ${ }^{* *} p$-value $<0.001$. (E) Scheme of the targeting construct pROSA26-ERT2CreERT2 for the ROSA26 locus with the tamoxifen-inducible Cre recombinase (Matsuda \& Cepko, 2007). ERT2-Cre-ERT2 expression is under the control of the synthetic cytomegalo-virus/chicken-actin/beta-globin promoter (CAG). Homology arms for ROSA26 targeting are indicated in light grey. A blasticidin resistance cassette (BlaR) for positive selection is under the control of the SV40 promoter and terminator. (F) Predicted restriction pattern of ROSA26 WT and integration events (top) and fragments detected by Southern blotting (bottom). Left: The hybridization probe (P1) locating to the left ROSA26 homology arm detected a genomic fragment of $6.9 \mathrm{~kb}$. The $4.4 \mathrm{~kb}$ fragment represents the wild-type ROSA26 allele. Right: Detection of possible off-target events using a second probe (P2) locating to the blasticidin selection marker within the targeting construct. See Underlying data for the raw data behind this figure (Schwarz, 2020).

functional in complementing the developmental defects and lethality of homozygous $T d g^{\text {tmlPsch }} \mathrm{KO}$ embryos (Cortázar et al., 2011; Cortellino et al., 2011). MiniTdg complemented mice were healthy and fertile with normal lifespan.

Three ESC populations were then derived from male blastocysts from crosses of miniTdg $g^{t g / t} / / T d g^{-/-}$with $T d g^{+/-}$mice (Extended data, Table S2: Mm11, Mm01, Mm02) (Schwarz, 2020). In these
ESCs, TDG protein levels were similar to those in wild-type ESCs (Figure 1C), indicating that the miniTdg gene is regulated as the endogenous $T d g$. Furthermore, no silencing of the transgene was noticed over several generations of animal breeding or culturing of ESCs. To assess functional integrity at the molecular level, we measured the accumulation of oxidized 5-methylcytosine (5-mC) derivates, 5-hydroxymethylcytosine (5-hmC) and 5-carboxylcytosine (5-caC), the substrate for 
excision by TDG (Maiti \& Drohat, 2011; Shen et al., 2013) (Figure 1D). Mass spectrometry showed that TDG deficient ESCs accumulated 5-caC as expected, while ESCs expressing TDG solely from the miniTdg gene showed 5-caC levels similar to those in wild-type ESCs.

To allow for a controlled excision of the miniTdg coding region, we introduced a tamoxifen-inducible Cre recombinase expression cassette (Figure 1E) into the non-essential ROSA26 locus (Soriano, 1999). Screening 60 blasticidin-resistant colonies by PCR for targeted integration at 3' ROSA26 locus yielded six clones (TDGiKO1-6). Southern blotting confirmed correct heterozygous integration of the complete $\mathrm{ER}^{\mathrm{T} 2}-\mathrm{Cre}-\mathrm{ER}^{\mathrm{T} 2}$ cassette (Matsuda \& Cepko, 2007) for four of the six clones (Figure 1F, left, asterisks). Potential additional off-target integrations were tested and excluded by hybridizing a second probe within the transgene (Figure 1F, right). Original Southern blotting images are available as Underlying data (Schwarz, 2020).

Taken together, we established a miniTdg gene that complements expression and function of the endogenous $T d g$ gene in vivo and in vitro. The functional integrity of the transgene is confirmed by rescuing phenotypes of $T d g \mathrm{KO}$ defects at the systemic and cellular level, namely the rescue of embryonic lethality and suppression of 5-caC accumulation. The introduction of a Cre-recombinase to the ROSA26 locus, without off-targeted events, will allow conditional inactivation of the miniTdg in ESCs.

In addition, we generated ESC clones that ease further genetic manipulations involving selectable marker genes. We eliminated the neomycin resistance gene, which was introduced at the endogenous $T d g$ locus to generate the original $T d g \mathrm{KO}$ allele (Kunz et al., 2009), by applying a CRISPR-guided Cas9 nuclease. Using two guide RNAs targeting the 5' and 3' end of the $N e o^{R}$ gene, we generated two ESC clones (TDGiKO1.1 and TDGiKO1.2) that bear a deletion of 776 and $767 \mathrm{bp}$, respectively, and are no longer resistant to neomycin (Extended data, Figure S1A-C) (Schwarz, 2020).

\section{Induction of the Cre recombinase leads to fast and efficient depletion of TDG in ESCS}

To make the inducible TDG depletion (Figure 2A) applicable to differentiation experiments that often depend on complex and strict cell culture procedures, we aimed at establishing a short Cre-induction procedure minimizing any interference with standard differentiation conditions. Titrating 4-Hydroxytamoxifen (OHT) and measuring miniTdg excision by quantitative PCR, we identified OHT concentrations of $1-5 \mu \mathrm{M}$ in serum-free $2 \mathrm{i}$ (Figure 2B) and 5-10 $\mu \mathrm{M}$ OHT in serum-containing medium (Figure 2C) to yield maximal excision efficiencies without impairing cell viability (Figure 2D). We note that lower OHT doses (down to $100 \mathrm{nM}$ ) for longer duration (up to 48 h) may be applied as well, depending on the experimental procedure and objective. While the miniTdg excision product is readily detectable by PCR in different TDGiKO ESCs after OHT treatment, no unintentional background activity of the Cre recombinase is detectable before OHT induction
(Figure 2E). Following the kinetics of TDG depletion by SDS-PAGE/immuno-detection, we found that TDG was reduced below detection limit ( $<2 \%$ of endogenous TDG) $24 \mathrm{~h}$ after Cre induction for $2 \mathrm{~h}$ (Figure $2 \mathrm{~F}$ and Extended data, Figure S1D) (Schwarz, 2020).

We also assessed the tissue specific induction of Cre-mediated miniTdg excision in vivo. We crossed miniTdg mice with mice expressing Cre-recombinase under the control of a FoxN1-promoter (http://www.informatics.jax.org/allele/key/62500) which drives expression in thymic epithelial cells (TECs), but not in thymic cells (TCs). We observed that the mRNA and protein expressed from the miniTdg are reliably depleted in TECs expressing FoxN1-driven Cre, whereas TDG levels are much less affected in TCs (Extended data, Figure S1E/F) (Schwarz, 2020).

These data demonstrate the functionality and tight regulation of the inducible miniTdg KO model in murine ESCs as well as in mice. A short-time Cre induction with $1-5 \mu \mathrm{M}$ or $5-10 \mu \mathrm{M}$ OHT (in $2 \mathrm{i}+\mathrm{LIF}$ or $15 \%$ FCS-containing medium, respectively) is sufficient to mediate excision of the miniTdg, resulting in depletion of TDG below detection within 24 hours. This rapid depletion of TDG is presumably facilitated by the cell cycleregulated proteasomal degradation of TDG via the ubiquitination at its PCNA-interacting peptide (PIP) motif (Hardeland et al., 2007; Shibata et al., 2014).

\section{Molecular and cellular phenotypes of TDG depletion in TDGiKO ESCS}

To validate the molecular phenotype of TDG depletion in our newly derived TDGiKO ESCs (TDGiKO1 ESCs and derived $\mathrm{Neo}^{\mathrm{R}}$-deleted TDGiKO1.1), we again assessed changes in levels of oxidized 5-mC derivates, 5-formylcytosine (5-fC) and 5-caC following Cre-mediated miniTdg deletion. One week after induction, we measured a 4.4 and 4.2-fold accumulation of the TDG substrates 5-fC and 5-caC in TDG-depleted TDGiKO ESCs, respectively, but no changes of 5-hydroxymethyl cytosine $(5-\mathrm{hmC})$, which is not recognized by TDG (Figure 3A). This accumulation reflects well the previously reported measurements in constitutive $T d g \mathrm{KO}$ cells (Shen et al., 2013; Steinacher et al., 2019).

Finally, we re-tested the pluripotency and differentiation capacity of some of the engineered TDGiKO ESC clones (Extended data, Table S2) (Schwarz, 2020), applying a protocol for alltrans retinoic acid (RA) induced in vitro differentiation towards the neuronal lineage (Bibel et al., 2007) with minor adaptations. TDG depletion was induced just before an additional culturing period of $16 \mathrm{~h}$ in serum containing medium, which promotes the transition from the ground (cultured in $2 \mathrm{i}$ medium) to the primed ESC state (Figure 3B). Formation of embryoid bodies and neural progenitor cells was triggered by LIF withdrawal and later the addition of RA, before terminal differentiation was induced by replating the cells in media for neural cells. When subjecting TDG proficient TDGiKO1 ESCs to neural differentiation, we observed an upregulation of the neural marker Nestin (Figure 3B, C) and, ultimately, the formation of neuron-like cells (Figure 3B). As expected during embryoid body (EB) formation, 
A

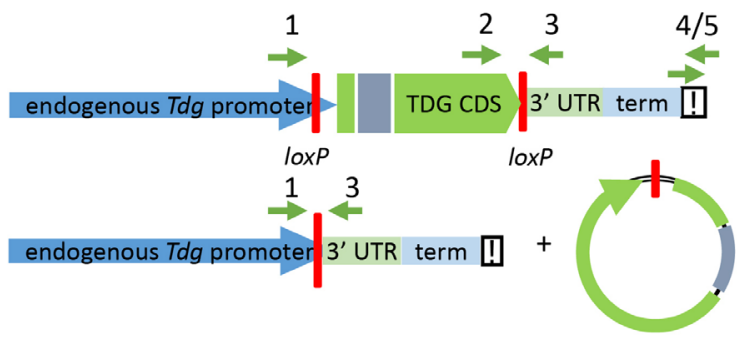

B
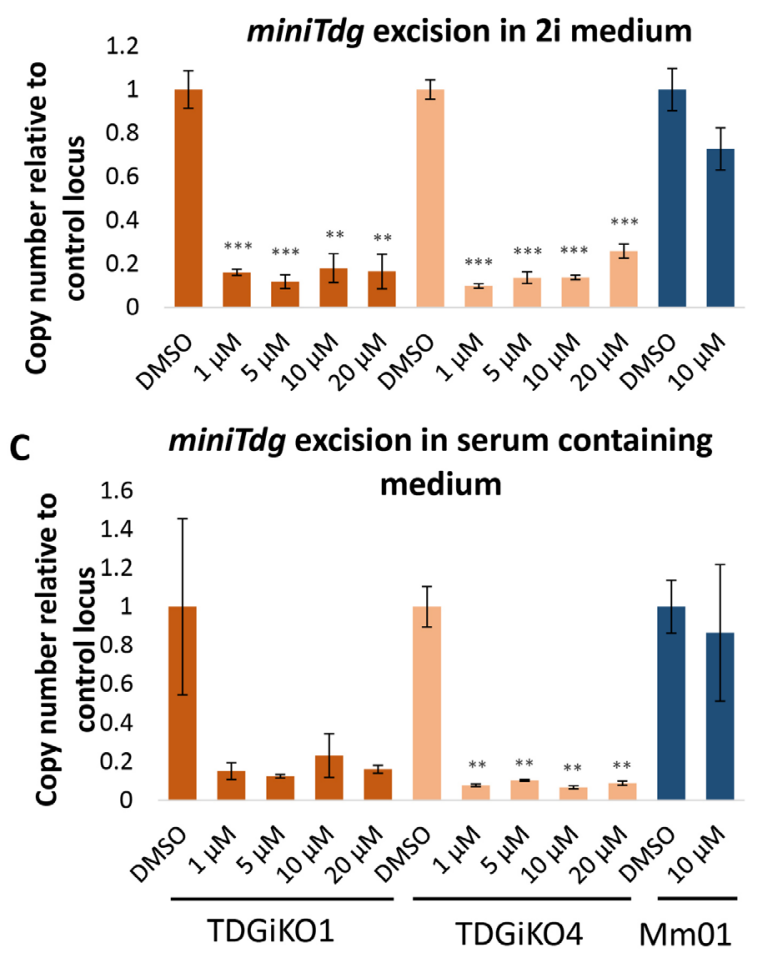

D

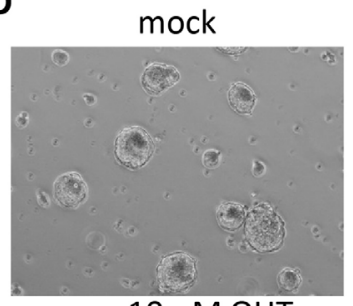

$10 \mu \mathrm{M} \mathrm{OHT}$

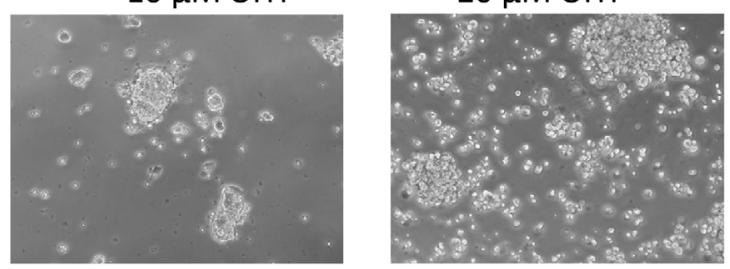

E $\mathrm{H}_{2} \mathrm{OMm01}$ TDGiKO1 TDGiKO4 TDGiKO1.1

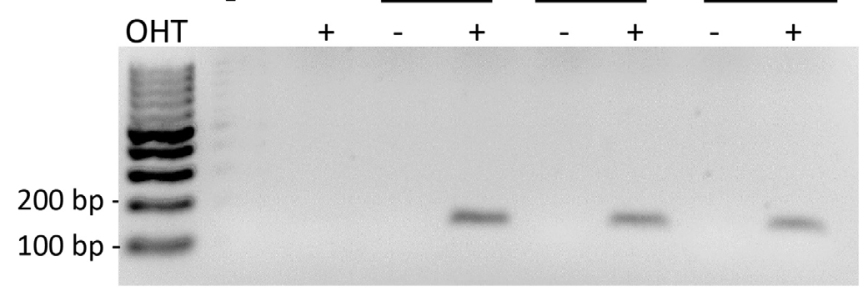

$\mathbf{F}$

Time after induction [hrs]

TDG

Figure 2. Cre induction for two hours results in the depletion of TDG within $\mathbf{2 4}$ hours without affecting cell viability. (A) Scheme of the miniTdg cassette before (top) and after (bottom) Cre-mediated recombination. Position of PCR primers are indicated with green arrows. (B) Quantitation of miniTdg excision upon OHT administration in TDGiKO1, TDGiKO4 and parental ESCs without the Cre recombinase (Mm01). Genomic DNA of cells was extracted two days after OHT treatment with indicated concentrations for 2 hours. Copy number of the miniTdg is measured by GPCR using primers 2 and 3, normalized to the nearby terminator region (primers 4/5) and a control locus on chromosome two. Shown are means and standard error from technical quadruplicates per clone. Asterisks indicate $p$-values of Student's t-test, compared to DMSO control: ** $p$-value $<0.01, * * * p$-value $<0.001$. (C) Quantitation of miniTdg excision as in (B), but Cre induction executed in ES medium containing 15\% FCS. (D) Phase-contrast images of TDGiKO1 ESCS in 2i medium, two days after OHT treatment for two hours at indicated concentrations. (E) Detection of Cre recombination events (158 bp) by PCR using primers 1 and 3 (A) before and after addition of OHT. (F) Time-course assessment of TDG protein levels expressed from the miniTdg gene. Immunodetection with an anti-TDG antibody was performed with western blotted NP-40 cell extracts of $2 \mathrm{i}$ cultivated TDGiKO1 ES cells after Cre induction by $1 \mu \mathrm{M}$ OHT for $2 \mathrm{~h}$. See Underlying data for the raw data behind this figure (Schwarz, 2020).

LIF withdrawal resulted in an induction of lineage-specific genes of all three germ layers and the repression of the pluripotency marker gene Nanog. This indicates the ability of the TDG proficient TDGiKO ESCs to commit to cell types of all germ layers. In support of this, continued cultivation of non-induced TDGiKO1 and TDGiKO1.1 in ESM without LIF led to a spontaneous differentiation to cardiomyocytes (mesodermal cell type) forming beating organoids (see Extended data Videos 1 and 2) (Schwarz, 2020). While the early initiation of lineage-specific genes seemed to be unaffected by TDG depletion prior to differentiation, the formation of neuron-like cells (Figure 3B, bottom) was impaired. This deficiency to stably commit to and/or maintain the neuronal lineage was indicated by a low number of neuron-like cells formed and the loss of cells with Nestin expression following RA application. Upon loss of TDG, we furthermore observed a significant difference in the downregulation of the pluripotency marker Nanog as well as and dysregulation of the endodermal marker Gata6 (Figure 3C). 
A
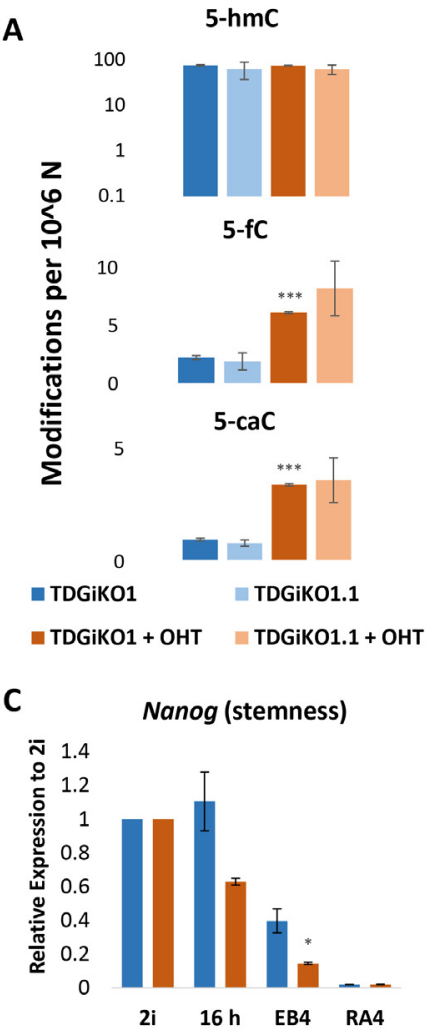

B

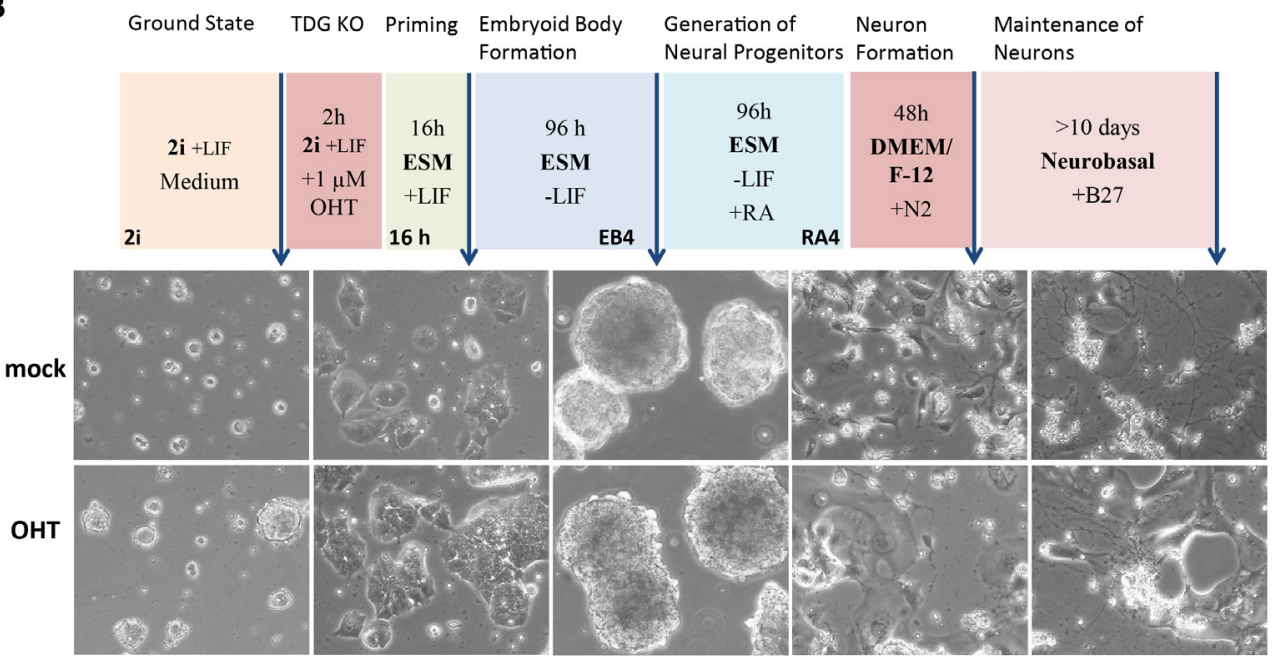

Nestin (ectoderm)

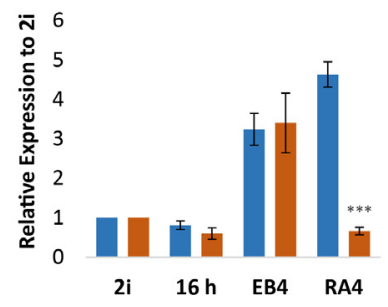

Bmp4 (mesoderm)

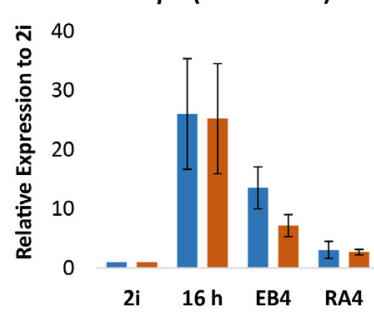

Gata6 (endoderm)

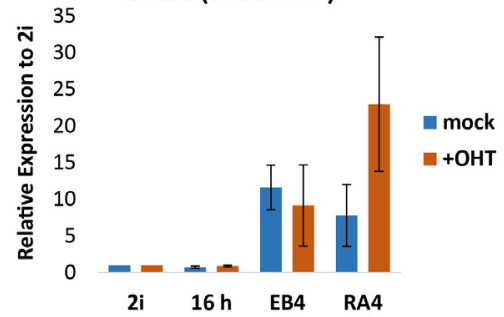

Figure 3. TDGiKO ESCs express a functional TDG and are proficient for cell lineage commitment. (A) Measurement of oxidized derivates of 5-mC in selected ESCs by HPLC-MS/MS, cultivated in 2i medium + LIF, one week after TDG depletion by $1 \mu$ M of OHT for 2 h. Shown are the means and standard deviation of 2-3 biological replica per condition. (B) Scheme of the neural differentiation protocol (Bibel et al., 2007), adapted with a $16 \mathrm{~h}$ priming step in ESC medium for ESCS grown in $2 \mathrm{i}$ medium. Representative phase-contrast images of TDGiKO1 cells with (+OHT) and without (mock) TDG depletion at the differentiation stages indicated (arrows). (C) Expression of pluripotency and germ layer marker genes were assessed by qRT-PCR in TDGiKO1 cells at the stages of differentiation as marked in (B). Expression was normalized to the housekeeping genes Rps13 and Eef1a1. Shown are means and standard errors of 3 biological replicates. Asterisks indicate $p$-values of Student's t-test, compared to the non-induced condition: ${ }^{*} p$-value $<0.05, * * \star p$-value $<0.001$. See Underlying data for the raw data behind this figure (Schwarz, 2020).

Thus, when subjecting the TDG-depleted TDGiKO ESCs to in vitro differentiation, we obtained data consistent with previous observations in constitutive Tdg KO ESCs (Steinacher et al., 2019). This highlights the usability of the newly established ESCs in TDG and BER-related research, with the advantage of eliminating clonal divergences between TDG proficient and deficient cells. While here, we focused on the reproduction of published $T d g$ KO phenotypes, the fast and reliable depletion of TDG in the TDGiKO ESC model now provides a tool for in-depth execution point analysis of TDG-BER mediated active DNA demethylation during cell differentiation or in any other context of interest. Furthermore, the loxP sites flanking the entire coding region of the miniTdg gene enable recombinase-mediated cassette exchange to introduce TDG separation of function variants devoid of catalytic function or harbour mutations that ablate posttranslational modifications (Hardeland et al., 2000). Such an exchange with functional TDG variants or fusion proteins is not easily applicable with other available murine conditional Tdg knockout systems (e.g.: Cortellino et al., 2011; Hassan et al., 2017; Hu et al., 2014; Song et al., 2013). Additionally, the availability of mice carrying the miniTdg, which can be combined with Cre-driver mice of interest by crossing, allows the translation of in vitro observations into in vivo settings. All in all, we provide a versatile, well-controlled and validated toolbox (Extended data, Table S2) (Schwarz, 2020) to facilitate functional and mechanistic research on TDG-mediated active DNA demethylation and BER in cell culture and animal models.

\section{Data availability}

Underlying data

Zenodo: Inducible TDG knockout models to study epigenetic regulation. http://doi.org/10.5281/zenodo.4075154 (Schwarz, 2020).

This project contains the following underlying data:

- Raw_Data_Figure1.zip. Database excerpt for genotyping of mouse crossings, original pictures of Southern and western blots and MS-quantification of oxidized $\mathrm{mC}$ derivates (also for figure 3).

- Raw_Data_Figure2.zip. qPCR data of miniTdg excision, original pictures of OHT-treated ESCs and western blots. 
- Raw_Data_Figure3.zip. Original pictures of differentiating cells and qPCR data of pluripotency and germ line markers.

- Raw_Data_Extended_Figure.zip. Sequencing data for $N e o^{R}$ excision, and predicted off-targets, absorption values of G418-treated ESCs, qPCR data for miniTdg excision and original pictures of western blots.

\section{Extended data}

Zenodo: Inducible TDG knockout models to study epigenetic regulation. http://doi.org/10.5281/zenodo.4075154 (Schwarz, 2020).

This project contains the following extended data:

- Extended Figure and Tables.pdf. Supplementary information as referenced in the text.

- Extended_Video_1_TDGiKO1.mp4. Video of "beating bodies" from an ESC clone.
- Extended_Video_2_TDGiKO1.2.mp4. Video of "beating bodies" from a second ESC clone.

Data are available under the terms of the Creative Commons Attribution 4.0 International license (CC-BY 4.0).

\section{Acknowledgments}

We want to express our thanks to the laboratory of Wolf Reik and the transgenics facility at the Babraham Institute (Cambridge, UK) for performing the pronuclear injection. We also thank the animal facility at the DBM Mattenstrasse and the transgenic core facility of the University of Basel for their help in breeding the mouse strains and establishing ESCs. Additional thanks goes to Carlos Mayer from George Holländer's Lab (DBM) for the sorting of the thymic cells.
Bibel M, Richter J, Lacroix E, et al.: Generation of a defined and uniform population of CNS progenitors and neurons from mouse embryonic stem cells. Nat Protoc. 2007; 2(5): 1034-1043. PubMed Abstract | Publisher Full Text

Cortázar D, Kunz C, Selfridge J, et al:: Embryonic lethal phenotype reveals a function of TDG in maintaining epigenetic stability. Nature. 2011; 470(7334): 419-423.

PubMed Abstract | Publisher Full Text

Cortellino S, Xu J, Sannai M, et al.: Thymine DNA glycosylase is essential for active DNA demethylation by linked deamination-base excision repair. Cell. 2011: 146(1): 67-79.

PubMed Abstract | Publisher Full Text | Free Full Text

Hardeland $\mathrm{U}$, Bentele $\mathrm{M}$, Jiricny J, et al.: Separating substrate recognition from base hydrolysis in human thymine DNA glycosylase by mutational analysis. J Biol Chem. 2000; 275(43): 33449-33456.

PubMed Abstract | Publisher Full Text

Hardeland U, Kunz C, Focke F, et al.: Cell cycle regulation as a mechanism for functional separation of the apparently redundant uracil DNA glycosylases TDG and UNG2. Nucleic Acids Res. 2007; 35(11): 3859-3867.

PubMed Abstract | Publisher Full Text | Free Full Text

Hassan HM, Kolendowski B, Isovic M, et al.: Regulation of Active DNA Demethylation through RAR-Mediated Recruitment of a TET/TDG Complex. Cell Rep. 2017; 19(8): 1685-1697.

PubMed Abstract | Publisher Full Tex

He YF, Li BZ, Li Z, et al.: Tet-mediated formation of 5-carboxylcytosine and its excision by TDG in mammalian DNA. Science. 2011; 333(6047): 1303-1307.

PubMed Abstract | Publisher Full Text | Free Full Text

Henry RA, Mancuso P, Kuo YM, et al.: Interaction with the DNA Repair Protein Thymine DNA Glycosylase Regulates Histone Acetylation by p300. Biochemistry. 2016; 55(49): 6766-6775.

PubMed Abstract | Publisher Full Text | Free Full Text

Hu X, Zhang L, Mao SQ, et al.: Tet and TDG mediate DNA demethylation essential for mesenchymal-to-epithelial transition in somatic cell reprogramming. Cell Stem Cell. 2014; 14(4): 512-22.

PubMed Abstract | Publisher Full Text

Ito $\mathrm{S}$, Shen $\mathrm{L}$, Dai Q, et al.: Tet proteins can convert 5-methylcytosine to 5 formylcytosine and 5-carboxylcytosine. Science. 2011; 333(6047): 1300-1303. PubMed Abstract | Publisher Full Text | Free Full Text

Jacobs AL, Schär P: DNA glycosylases: In DNA repair and beyond.

Chromosoma. 2012; 121(1): 1-20.

PubMed Abstract | Publisher Full Text | Free Full Text

Kunz C, Focke F, Saito $Y$, et al.: Base excision by thymine DNA glycosylase mediates DNA-directed cytotoxicity of 5-fluorouracil. PLOS Biol. 2009; 7(4): e91.

PubMed Abstract | Publisher Full Text | Free Full Text

Léger H, Smet-Nocca C, Attmane-Elakeb A, et al.: A TDG/CBP/RARa ternary complex mediates the retinoic acid-dependent expression of DNA methylation-sensitive genes. Genom Proteom Bioinf. 2014; 12(1): 8-18. PubMed Abstract | Publisher Full Text | Free Full Text

Maiti A, Drohat AC: Thymine DNA glycosylase can rapidly excise

5-formylcytosine and 5-carboxylcytosine: potential implications for active demethylation of CpG sites. J Biol Chem. 2011; 286(41): 35334-35338. PubMed Abstract | Publisher Full Text | Free Full Text

Matsuda T, Cepko CL: Controlled expression of transgenes introduced by in vivo electroporation. Proc Natl Acad Sci U S A. 2007; 104(3): 1027-1032. PubMed Abstract | Publisher Full Text | Free Full Text

Schuermann D, Weber AR, Schär P: Active DNA demethylation by DNA repair: Facts and uncertainties. DNA Repair (Amst). 2016; 44: 92-102.

PubMed Abstract | Publisher Full Text

Schwarz SD: Inducible TDG knockout models to study epigenetic regulation [Data set]. F1000 Research. Zenodo. 2020.

http://www.doi.org/10.5281/zenodo.4075154

Shen L, Wu H, Diep D, et al.: Genome-wide analysis reveals TET- and TDGdependent 5-methylcytosine oxidation dynamics. Cell. 2013; 153(3): 692-706.

PubMed Abstract | Publisher Full Text | Free Full Text

Shibata E, Dar A, Dutta A: CRL4Cdt2 E3 ubiquitin ligase and Proliferating Cell Nuclear Antigen (PCNA) cooperate to degrade thymine DNA glycosylase in S phase. J Biol Chem. 2014; 289(33): 23056-23064.

PubMed Abstract | Publisher Full Text | Free Full Text

Song CX, Szulwach KE, Dai Q, et al.: Genome-wide profiling of

5-formylcytosine reveals its roles in epigenetic priming. Cell. 2013; 153(3): $678-91$.

PubMed Abstract | Publisher Full Text | Free Full Text

Soriano P: Generalized lacZ expression with the ROSA26 Cre reporter strain. Nat Genet. 1999; 21(1): 70-71.

PubMed Abstract | Publisher Full Tex

Steinacher R, Barekati Z, Botev P, et al.: SUMOylation coordinates BERosome assembly in active DNA demethylation during cell differentiation. $E M B O$ f. 2019; 38(1): e99242.

PubMed Abstract | Publisher Full Text | Free Full Text

Um S, Harbers M, Benecke A, et al.: Retinoic Acid Receptors Interact Physically and Functionally with the T:G Mismatch-specific Thymine-DNA Glycosylase.J Biol Chem. 1998: 273(33): 20728-36. PubMed Abstract | Publisher Full Text

Weber AR, Krawczyk C, Robertson AB, et al.: Biochemical reconstitution of TET1-TDG-BER-dependent active DNA demethylation reveals a highly coordinated mechanism. Nat Commun. 2016; 7: 10806. PubMed Abstract | Publisher Full Text | Free Full Text Ying Q, Wray J, Nichols J, et al.: The ground state of embryonic stem cell selfrenewal. Nature. 2008; 453(7194): 519-524.

PubMed Abstract | Publisher Full Text | Free Full Text 


\section{Open Peer Review}

\section{Current Peer Review Status:}

\section{Version 2}

Reviewer Report 02 December 2020

https://doi.org/10.5256/f1000research.30222.r73317

(c) 2020 Bultmann S. This is an open access peer review report distributed under the terms of the Creative Commons Attribution License, which permits unrestricted use, distribution, and reproduction in any medium, provided the original work is properly cited.

\section{Sebastian Bultmann}

Department of Biology II and Center for Integrated Protein Science Munich (CIPSM), LudwigMaximilians-Universität München, Munich, Germany

The authors have addressed all concerns.

Competing Interests: No competing interests were disclosed.

Reviewer Expertise: Epigenetics, embryonic stem cells, gene editing

I confirm that I have read this submission and believe that I have an appropriate level of expertise to confirm that it is of an acceptable scientific standard.

\section{Version 1}

Reviewer Report 07 October 2020

https://doi.org/10.5256/f1000research.28295.r72019

(c) 2020 Bultmann S. This is an open access peer review report distributed under the terms of the Creative Commons Attribution License, which permits unrestricted use, distribution, and reproduction in any medium, provided the original work is properly cited.

\section{Sebastian Bultmann}

Department of Biology II and Center for Integrated Protein Science Munich (CIPSM), LudwigMaximilians-Universität München, Munich, Germany

In the manuscript entitled "Inducible TDG knockout models to study epigenetic regulation" the authors present new model system for the conditional depletion of TDG. The authors employ a 
strategy in which a loxP flanked mini gene is introduced into a TDG KO background. In combination with an inducible Cre introduced into the ROSA26 locus the authors convincingly show the rapid end efficient depletion of TDG. Finally, the authors validate their system by investigating the effect of acute TDG depletion on differentiation.

In summary, the study presents a clever strategy for induced deleption, is well thought through and scientifically sound.

I have only two minor points the authors should address:

1. Is the TDG mini gene randomly integrated into the genome? The authors should make this clear and if possible comment on any observation regarding silencing of the transgene during long-term culture.

2. The section "molecular and cellular phenotypes" is a little confusing. The authors should make clear in the text when they are talking about induced (TDG depeltion) or uninduced (control) cells. The authors should clearly explain the experimental setup shown in 3B in the text. In particular in the second paragraph of this section the authors start describing the control cells and then suddenly about TDG depleted cells.

Is the rationale for developing the new method (or application) clearly explained? Yes

Is the description of the method technically sound?

Yes

Are sufficient details provided to allow replication of the method development and its use by others?

Yes

If any results are presented, are all the source data underlying the results available to ensure full reproducibility?

Yes

Are the conclusions about the method and its performance adequately supported by the findings presented in the article?

Yes

Competing Interests: No competing interests were disclosed.

Reviewer Expertise: Epigenetics, embryonic stem cells, gene editing

I confirm that I have read this submission and believe that I have an appropriate level of expertise to confirm that it is of an acceptable scientific standard.

Author Response 12 Oct 2020

David Schürmann, University of Basel, Basel, Switzerland 
We thank Dr Bultmann for the evaluation of our manuscript. We appreciate his comments helping to improve it. Accordingly, the following adaptations were made :

1. The first point was also raised by Dr Torchia (reviewer 2 ) and we describe better the random integration of the miniTdg gene. We also added a note about the transcriptional stability of the transgene in chapter 1 of the Result section.

2. We apologize for the confusing description of our results. We now clearly indicate whether we refer to TDG proficient or deficient cells. We also added an outline of the experimental setup for the neural differentiation.

Competing Interests: No competing interests were disclosed.

Reviewer Report 30 September 2020

https://doi.org/10.5256/f1000research.28295.r71089

(c) $\mathbf{2 0 2 0}$ Torchia J. This is an open access peer review report distributed under the terms of the Creative Commons Attribution License, which permits unrestricted use, distribution, and reproduction in any medium, provided the original work is properly cited.

\section{Joseph Torchia}

Department of Biochemistry, Western University, London, ON, Canada

Thymine DNA Glycosylase (TDG) is a BER protein that is essential for the removal of 5formylcytosine (5-fC) and 5-carboxylcytosine (5-fC) from genomic DNA. TDG knockout is embryonic lethal at E11.5 due to genome wide epigenetic instability. To circumvent embryonic lethality from a deletion of $T d g$, the authors synthesized a mini-Tdg allele consisting of an optimized TDG codon arrangement for expression of two naturally occurring TDG splice variants. The authors introduced the mini- $T d g$ allele in a $T d g^{-/-}$background to generate miniTdgtg/tg/Tdg ${ }^{-/}$mice, which phenocopy and express TDG similarly to wild-type mice. Furthermore, the TDG codons are flanked by loxp sites to allow for efficient spatial and temporal excision of $T d g$ in response to tamoxifen. The deletion of TDG in ESC's results in a global upregulation in 5-caC, and Tdg null ESC's fail to differentiate in response to retinoic acid both of which are consistent with previous literature. Overall, the paper describes a novel mouse model to study the role of TDG dependent active DNA demethylation in vitro and in vivo.

The paper is well written, and the data seems to be convincing. In addition, the experiments appear to be performed to a high standard. However, I feel addressing the following concerns would significantly strengthen the manuscript and increase the novelty of the mouse model:

\section{Major Comments:}

1. The authors need to site appropriate literature and/or provide data to address the claim that epigenetic instability resulting from a loss of TDG leads to complications in long-term ESCs cultures. Multiple studies, such as Hu et al. 2014 (PMID 24529596) and Shen et al. 2013 ${ }^{2}$ (PMID 23602152), have rigorously used $T d g^{\%}$ mouse ESC's without any mention of such complications. I feel the argument that the use of such a complicated system to study TDG 
deletion in MEFs and ESCs needs further clarification.

2. There are several published conditional knockout mouse models of TDGthat incorporate loxp sites in the endogenous $T d g$ allele. These mouse models, and their potential drawbacks, need to be discussed to provide a stronger argument for the use of mini-Tdg allele approach employed in this manuscript.

3. Its unclear why it was necessary to remove the neo cassette to generate the TDGiKO1.1 and TDGiKO1.2 ESC clones? The use of CRISPR can introduce complications such as off-target mutations and clonal selection that can introduce variability in the model.

Minor comments and questions:

1. Figure 1D, 2B, 2C, 3A are all lacking statistical analysis. Please use the appropriate nomenclature to indicate statistical significance.

2. Please describe the sample size for LC-MS/MS experiment outlined in figure 1D and Figure $3 \mathrm{~A}$.

3. Figure 2F: Why is TDG blot not showing a doublet. The SUMOylated TDG band is missing.

4. Why was 4-OHT employed at such a high concentration? In my opinion $100 \mathrm{nM}$ to $500 \mathrm{nM}$ should be more than sufficient to induce excision.

5. Was the mini-Tdg allele integrated in the mouse genome? If so, where did the integration occur?

\section{References}

1. Hu X, Zhang L, Mao SQ, Li Z, et al.: Tet and TDG mediate DNA demethylation essential for mesenchymal-to-epithelial transition in somatic cell reprogramming.Cell Stem Cell. 2014; 14 (4): 512-22 PubMed Abstract | Publisher Full Text

2. Shen L, Wu H, Diep D, Yamaguchi S, et al.: Genome-wide analysis reveals TET- and TDGdependent 5-methylcytosine oxidation dynamics.Cell. 2013; 153 (3): 692-706 PubMed Abstract I Publisher Full Text

Is the rationale for developing the new method (or application) clearly explained? Partly

Is the description of the method technically sound?

Yes

Are sufficient details provided to allow replication of the method development and its use by others?

Yes

If any results are presented, are all the source data underlying the results available to ensure full reproducibility?

Partly 
Are the conclusions about the method and its performance adequately supported by the findings presented in the article?

Yes

Competing Interests: No competing interests were disclosed.

Reviewer Expertise: Epigenetics, Biochemist

I confirm that I have read this submission and believe that I have an appropriate level of expertise to confirm that it is of an acceptable scientific standard.

Author Response 12 Oct 2020

David Schürmann, University of Basel, Basel, Switzerland

We thank Dr Torchia for the critical reading of our manuscript and his valuable suggestions for improvement, which helped a lot to strengthen our conclusions. We revised the manuscript according to his major and minor comments.

Major comments:

1. We understand the reviewers comment that this statement is rather speculative and currently poorly supported by data. We therefore removed it in the revised version. On the other hand, we observed in our experiments with constitutive TDG KO ESCS subtle and rather global differences (e.g. cell division time), which we do not yet fully understand. They could originate from clonal adaptation but also from TDG-driven epigenetic mechanisms. However, to be able to discriminate between these possibilities was the reason for the development of the here presented inducible TDG depletion system. Apart from the not yet ruled out role of TDG as repair enzymes for damaged DNA, the increase of $\mathrm{fC}$ and $\mathrm{caC}$ might affect DNA-protein interactions and consequently chromatin landscaping. Irrespective of these hypothetical scenarios, where long-term culturing of cells could result in both a genetic as well as an epigenetic drift, we feel that an isogenic system is always the preferable experimental system, even when it seems to be more complicated and laborious.

2. We are aware that several other conditional $T d g$ allele were generated and available as well, which are genetically easier to handle and could perfectly serve their experimental purposes. In fact, before having started to engineer our complementing miniTdg system, we intended but failed to introduce loxP sites at the extremities of the endogenous $T d g$ coding region to be able to delete the entire gene, omitting putative dominant truncated transcripts. We suspect that besides a poor accessibility of some regions of the $T d g$ gene, the multitude of pseudogenes consisting basically of $T d g$ cDNA sequences interferes with successful genome engineering in certain circumstances as they may act as off-targets, not only for classical gene targeting but also CRISPR/Cas9-based approaches. Having a functional miniTdg gene, in which the entire coding region is flanked by loxP sites, not only allows for an inducible TDG depletion but also enables further engineering steps in a flexible manner by Cre-mediated cassette exchange (although not designed specifically for this purpose). We refer to other available conditional $T d g$ allele in the revised version of our manuscript. In the last paragraph, furthermore, we point to 
this advantage and possibility. This was, by the way, one of the reasons why we deleted the neomycin resistance gene (see response to comment 3 below), allowing for the use of this selectable marker for such a replacement strategy.

3. We utterly agree with the reviewer about the potential drawbacks of the CRISPR/Cas9 genome editing approach. Therefore, we added our off-target analysis for the two ESC clones generated in the "Extended data".

In fact, most of the here presented data from cultured cells and in vivo are obtained from the systems without the additional deletion of the neomycin resistance gene (except LC-MS/MS data in Fig. 3A). For the sake of completeness of our system, we included these two ESC clones as they might be of interest when additional selection marker-based transgene integration is intended. These notions may have been presented ambiguously in the first version and are now improved by slightly rephrasing and restructuring of the respective paragraphs.

In any case, the possibility to work with the inducible TDG depletion systems ensures an isogenic background, at least for the role of TDG, which could be validated in different in ESC clones. Although one can never completely rule out some issues of clonal selection, we still consider it an advantageous approach.

Minor comments and questions:

1. We appreciate reviewer's pointing to the missing statistical indicators, which we now added.

2. More detailed information about the analysis by LC-MS/MS was added into the figure legends and the respective paragraph in the Methods section.

3. This is a very attentive comment by the reviewer. In contrast to Figure 1, indeed, the SUMO-modified TDG is not apparent in Figure 2F because we used NP-40 soluble protein extraction methods without the addition of specific inhibitors of SUMO proteases (f.e. N-Ethylmaleimide). As de-SUMOylation happens quite fast and efficiently during mild lysis condition even at $4^{\circ} \mathrm{C}$, SUMOylated TDG is in our hands only detectable when immediate lysis in Laemmli buffer and/or detergent-based lysis buffers supplement by the inhibitor. We now added the technical information about the extraction method also in the figure legend.

4. We agree with the reviewer that lower 4-OH tamoxifen concentration might work as well and added a comment about suitable Cre induction conditions in the $2^{\text {nd }}$ chapter of the results.

Based on an initial literature search, we found that commonly used concentrations of $\mathrm{OHT}$ are in the range of $100 \mathrm{~nm}-10 \mu \mathrm{M}$. To get a maximal flexibility and timely controlled TDG depletion in experimental protocols of differentiation, we aimed at rather short durations of induction. Such single induction 4-OHT treatments of as short as $2 \mathrm{~h}$ are rarely found in literature and associated with limited recombination efficiency (e.g. Buelow et al. 2008), while lower concentrations are more frequently used for Cre induction for up to $48 \mathrm{~h}$, sometimes also with repeated administration. For our purpose, we therefore opted for 4-OHT concentrations in the intermediate to high range but avoiding cytotoxic effects.

5. This is indeed a relevant point for a potential user of our system. The miniTdg gene was introduced by pronuclear injection of a plasmid that contained no homology arms for a targeted integration into the mouse genome. Thus, it is a random integration event as stated in the legend of Figure 1. We added now this information also the first result section. 
Unfortunately, our attempts to identify the genomic locus of integration by conventional molecular methods were so far not successful. Yet, we might get some hints from ongoing and future global NGS analyses, which would be amended to this paper as well as to the MGI entry.

Competing Interests: No competing interests were disclosed.

Reviewer Report 21 September 2020

https://doi.org/10.5256/f1000research.28295.r71086

(C) 2020 Sczepanski J. This is an open access peer review report distributed under the terms of the Creative Commons Attribution License, which permits unrestricted use, distribution, and reproduction in any medium, provided the original work is properly cited.

\section{Jonathan Sczepanski}

Department of Chemistry, Texas A\&M University, College Station, TX, USA

In the method article "Inducible TDG knockout models to study epigenetic regulation" Schwarz et. al establish mice and mESC cell line that allows for inducible KO of TDG. Building on their prior work, the key features of this system is a TDG mini-gene that resides between two loxP-Cre recombinase sequences, along with a tamoxifen-inducible Cre recombinase expression cassette. The authors demonstrate this design achieves near complete ( $98 \%)$ depletion of TDG upon tamoxifen-induction within $24 \mathrm{~h}$. Importantly, the TDG mini-gene recapitulates the epigenetic and repair functions of endogenous TDG prior to depletion, and cell morphology and pluripotency are not altered until after tamoxifen-induced depletion. These molecular and cellular phenotype studies are particularly well done, and clearly demonstrate agreement between inducible TDG KO ESCS and previously reported constitutive TDG KO ESCs. Overall, this work is very rigorous, and the controls are carefully designed and extensive. I anticipate that inducible TDG KO will be a valuable tool for mechanistic studies aimed at uncovering the role of TDG in DNA epigenetics and repair. I recommend this paper for indexing without further revisions.

Minor comment: ESC is written "ECS" in Figure 3 title.

Is the rationale for developing the new method (or application) clearly explained? Yes

Is the description of the method technically sound?

Yes

Are sufficient details provided to allow replication of the method development and its use by others?

Yes 
If any results are presented, are all the source data underlying the results available to ensure full reproducibility?

Yes

Are the conclusions about the method and its performance adequately supported by the findings presented in the article?

Yes

Competing Interests: No competing interests were disclosed.

Reviewer Expertise: DNA repair, base excision repair, DNA demethylation, thymine DNA glycosylase

I confirm that I have read this submission and believe that I have an appropriate level of expertise to confirm that it is of an acceptable scientific standard.

\section{Author Response 12 Oct 2020}

David Schürmann, University of Basel, Basel, Switzerland

We are very grateful to Dr Sczepanski for taking the time to evaluate our manuscript and were pleased to learn about his overall approval and appreciation of our experimental work and data.

The spelling mistake in the title of figure 3 was corrected.

Competing Interests: No competing interests were disclosed.

The benefits of publishing with F1000Research:

- Your article is published within days, with no editorial bias

- You can publish traditional articles, null/negative results, case reports, data notes and more

- The peer review process is transparent and collaborative

- Your article is indexed in PubMed after passing peer review

- Dedicated customer support at every stage

For pre-submission enquiries, contact research@f1000.com 